\title{
Depressive symptoms during adolescence: comparison between epidemiological and high risk sampling
}

\author{
Michelle C. St Clair • Ian M. Goodyer • \\ Valerie Dunn · Joe Herbert $\cdot$ Peter B. Jones • \\ Tim Croudace
}

Received: 23 June 2011 / Accepted: 11 October 2011/Published online: 30 October 2011

(C) The Author(s) 2011. This article is published with open access at Springerlink.com

\begin{abstract}
Purpose Both epidemiological (unselected) and high risk (screening on known risk criteria) samplings have been used to investigate the course of affective disorders. Selecting individuals on multiple risk criteria may create a sample not comparable to individuals with similar risk criteria within the general population. This study compared depressive symptoms across the two sampling methods to test this possibility.

Methods The high risk Cambridge Hormones and Moods Project (CHAMP) screened and recruited adolescents aged 12 to 16 . A total of 905 (710 high risk) individuals participated and were reassessed at three follow-ups. The ROOTS epidemiological sample consisted of 1,208 14-year-olds reassessed at 15.5 and 17 years. The risk profile for CHAMP was recreated in the ROOTS study. Both samples completed the Moods and Feelings Questionnaire, a self-report measure of current depressive symptoms.
\end{abstract}

M. C. St Clair $(\bowtie) \cdot$ I. M. Goodyer $(\bowtie) \cdot$ V. Dunn ·

T. Croudace

Department of Psychiatry, Developmental and Lifecourse

Research Group, University of Cambridge, Douglas House,

18b Trumpington Road, Cambridge CB2 8AH, UK

e-mail: mcs77@medschl.cam.ac.uk

I. M. Goodyer

e-mail: ig104@cam.ac.uk

J. Herbert

Department of Physiology, Development and Neurosciences,

Cambridge Centre for Brain Repair, University of Cambridge,

Cambridge, UK

P. B. Jones - T. Croudace

Department of Psychiatry, Addenbrookes Hospital,

University of Cambridge, Cambridge, UK
Results Comparing individuals with the same high risk profiles across the CHAMP and ROOTS studies revealed no significant differences in mean depression scores. Combining the samples revealed that for females, mean depression scores were maintained from 12 to 15 years then declined by 17 years. For males, scores declined from 12 throughout adolescence. High risk status led to consistently higher levels of depressive symptoms in female adolescents but result in little change within male adolescents.

Conclusions The high risk design recruited adolescents with a depression symptoms profile comparable to the general population for both sexes. High risk status may alter the trajectory of depressive symptoms in female adolescents only. Males may be less sensitive to recent adversity.

Keywords High risk - Epidemiological - Depressive symptoms · Trajectories

\section{Introduction}

To date, many psychosocial risk studies have used one risk criterion (present/absent) to select a sample "at risk" for increased mental health difficulties. These have included individuals with a previous history of depression [1], parental or grandparental psychiatric illness [2-4], parental substance abuse [5], poverty [6,7], parental divorce [8], or negative cognitive style [9]. This dichotomous approach is relatively simplistic and multiple risk criteria are likely to reveal a greater range and denote the complexities of risk influence on the development of psychopathology in young people [10-13].

Recent research has utilised multiple risk screening methodology to maximise the rate of depressive episodes 
observed in prospective cohort studies. This procedure combines a set of risk measures, each known to increase the liability of subsequent disorder. The method has captured a larger proportion of depressed cases by a factor of three over that of a standard sampling approach in a shortterm (12 months) follow-up [14, 15]. High risk in these studies was considered as either having a first degree relative with a history of psychiatric illness or two of the remaining risk criteria. Thus, positivity on a single risk criterion, excepting familial psychiatric illness, was not sufficient to classify as high risk, in contrast to previous studies $[1,5,6]$. Screening individuals in this manner may create samples with risk configurations that are not comparable to the wider population.

We tested the validity of generalising results from a high risk sample by comparing levels of depressive symptoms between two adolescent studies with similar risk profiles: one screened on multiple risk criteria, the other an epidemiological sample. We hypothesised that high risk individuals of either sex would have a similar level of depressive symptoms across the two sampling methods. We also tested a second hypothesis that the trajectory of depressive symptoms in high risk adolescents would be elevated over time and show less variation than expected in an epidemiological sample, indicating a more vulnerable group in the population at large. We further conjectured that females would show an elevated level of depressive symptoms compared to males.

\section{Method}

\section{Participants}

The Cambridge Hormones and Moods Project (CHAMP) $[16,17]$ screened a large sample of adolescents on particular depressogenic risks $[14,15]$. These were presence/ history of a psychiatric illness in a first degree family member, two bereavements (deaths or permanent separations) in the individual's lifetime, a negative emotional temperament, marital disharmony ( $>6$ months) or parental separation, two recent undesirable life events or chronic difficulties with family/friends (i.e., grandparent with dementia living within family long term). An individual was considered to be at higher risk if they had: (1) familial psychiatric illness, or (2) two or more of the remaining risk criteria.

A total of 3,677 children aged 12 to 16 from 11 Cambridgeshire secondary schools were screened. Of these, 1,970 were invited to participate and 905 individuals (age range: $12 ; 2-16 ; 11$ ) consented to the full study, with 710 at "high risk" of developing depression. The remaining 195 comparison participants were considered not high risk but were not necessarily without any risk criteria. The CHAMP participants were followed up at 4,8 and 12 months. The second and third measurements were postal questionnaires, whereas the first and fourth assessments were face-to-face interviews with questionnaires. There were 1,662 female and 2,015 male participants in the screening sample and 405 females and 500 males within the full study. Participants were only excluded if they had a current or a recent (within 3 months) mental illness.

The ROOTS study is a longitudinal investigation of an unselected, epidemiological sample of 14-year-olds (age range: 13;11-15;3), recruited from 18 East of England secondary schools by the same research team [18]. A total of 3,762 14-year-olds were invited into the study, 1,208 individuals consenting. There were 661 female and 547 male participants. Participants were followed up at 18 months with questionnaires and 36 months with faceto-face interviews and questionnaires.

During ROOTS baseline interviews, a similar evaluation of the risk criteria described above was performed. For life events and negative emotionality, the same measures were available in both samples. The remaining risk factors were derived from questionnaire data and information from a parental interview, the Cambridge Early Experience Interview (CAMEEI) [10].

Ethical approval was granted for the ROOTS study in accordance with the Declaration of Helsinki and Good Clinical Practice guidelines by Cambridgeshire 2 REC. At study entry, all participants and their parents gave written informed consent. Ethical approval for the CHAMP study was granted by the Cambridgeshire local ethics committee. For the screening procedure there was an "opt out" process with consent to be approached given by the return of the questionnaires. Written informed consent was subsequently obtained for all participants and their parents at first faceto-face meeting in the year-long study.

\section{Measures}

The self-report Moods and Feelings Questionnaire (MFQ) $[19,20]$ is a 33 item instrument that measures current (last 2 weeks) depressive symptoms in 8 to 18 -year-olds [19, 21]. We used a four category response version ("never", "sometimes", "mostly", and "always"). Due to the rarity of "always" responses, the "mostly" and "always" categories were combined. This measure was completed: (1) four times by the CHAMP participants at entry, 4,8 and 12 months; (2) three times by the ROOTS participants at entry, 18 and 36 months.

The EAS Temperament Questionnaire [22] was filled in by a parent/guardian (usually mother). This questionnaire assesses emotionality, activity, shyness and sociability over the lifetime of the child. From prior research [23], an 
individual was considered to have a "negative emotional temperament" if they scored above 17 on the emotionality scale sum score.

A parental questionnaire was used in both studies to record the presence of significant negative life events in the previous 12 months of the participants' life (i.e., changing school, moving home, or being hospitalised). Within the CHAMP study, parental questionnaires recorded any deaths or permanent separations over the course of the participants' lifetime as well as the perceived level of distress to the loss. Parental questionnaires also recorded family medical history, marital difficulties and chronic difficulties (defined as long term medical, financial or social difficulties) $[14,16]$.

The CAMEEI is an in-depth semi-structured parental interview that investigated the early life of ROOTS participants [10]. Questions relating to parental breakup, deaths/permanent separations and other chronic problems were taken from this interview and used as risk criteria. Familial psychiatric problems were measured from a parental questionnaire relating to current/past medical, emotional, or behavioural problems.

\section{Data analytic strategy}

We first investigated whether the prevalence of risk criteria used to stratify the samples was comparable across CHAMP and ROOTS before investigating whether the level of depressive symptoms in similar risk groups differed across the two studies. We used the entire CHAMP screening sample $(n=3,677)$ in the prevalence comparisons, which was restricted to only those who participated in the study when investigating the MFQ $(n=894)$.

When jointly analysing both studies, we equated individuals on age by investigating only those aged 14 years. For the ROOTS study, we used the baseline measurement. For the CHAMP study, we only included data reported by a 14-year-old participant. For individuals with more than one assessment during the relevant age-window, we used only the first measurement after they turned 14. This led to a final sample size for CHAMP of $n=1,300$ for the prevalence comparisons and $n=500$ for analysis with MFQ. We were unable to investigate only lower risk individuals as there were too few individuals within CHAMP.

\section{Prevalence comparisons}

To investigate prevalence differences in the risk criteria between the samples, logistic regressions were conducted with risk criterion present (1) versus absent (0) with a study dummy variable as the independent variable. When analysing the MFQ scores and risk criteria, regressions were conducted including gender as a covariate in addition to study main effects and interaction terms (risk criterion by study). The interaction terms determine whether the relationships between the MFQ scores and the risk criteria (present/absent) were similar in each study.

\section{Trajectory analyses}

We analysed cross-sectional age cohorts in CHAMP alongside the longitudinal data from the ROOTS study. Using age at entry, CHAMP participants were divided into four cohorts (12, 13, 14 and 15 years) and the MFQ measurement completed at study entry was used. We only used these baseline depression scores as there was a large panel conditioning effect evident by a substantial drop in average depression scores between baseline and 4 months. Panel effects were not observed in the ROOTS study, thus the CHAMP baseline measurement was considered most directly comparable to the ROOTS study.

\section{Multiple imputation}

The missing data within the ROOTS study underwent a multiple imputation procedure. We found that those who were missing at time 2 had higher MFQ scores at time 1 than those who were included, therefore, we decided to impute all missing data at all three time points. The imputation model contained all of the 33 MFQ items at each timepoint, as well as gender, SES (Acorn group), risk status, and the individual risk criteria. Gender, SES and risk status were also related to attrition across the longitudinal design. However, we restricted the imputation to only individuals with full data on the risk status and the individual risk criteria, as we did not want the model to impute these variables. Twenty imputations were created within the ice command in Stata [24]. Rubin's rules were used when combining the imputed datasets for analysis.

\section{Multilevel models}

The trajectories were analysed using multi-level mixedeffects linear regressions using maximum likelihood, which account for non-independence inherent within longitudinal data. The mim prefix was used to combine the multiply imputed datasets, which accounted for selective attrition. For the ROOTS data, a random intercept for ID was also included across to analyse the longitudinal data. We first tested for linear and quadratic differences in the trajectories. If these were significant, we further tested for categorical differences, using a categorical fixed effect. Age was entered as a categorical fixed effect for the CHAMP data. Analyses were stratified by gender, as there is generally a higher prevalence of depressive symptoms in 
female adolescents $[25,26]$. For the CHAMP data, male/ female differences within each age cohort were analysed with similar mixed-effects regressions. With the ROOTS data, gender differences were tested longitudinal using a gender by age interaction term.

The graphs were created using fitted values from the mixed-effects regressions. Fitted values (with corresponding standard errors) were used as they provided a single, averaged estimate across the 20 imputed datasets. All analyses were conducted within Stata/IC [24].

\section{Results}

Risk criteria at entry

The prevalence of risk criteria met in each study is shown in Table 1. There was an elevated prevalence in the CHAMP high risk study on each risk criterion with the exception of marital problems and chronic difficulties, which were elevated in the ROOTS epidemiological study. This pattern was replicated with only high risk individuals, except there were no differences within the familial psychiatric illness and high emotionality risk criteria.
Depressive symptoms

There was a higher level of depressive symptoms in the CHAMP compared to the ROOTS study (Table 2).

Comparing only those at high risk in both studies revealed no differences in mean depression scores. Similarly, there was no difference between the studies among those classified as not high risk. The study by risk status interaction term was not significant, $p=.44$, indicating that the relationship between high and not high risk was similar in both samples when looking at depressive symptoms.

Each of the individual risk criteria (with differing rates of prevalence across the two studies) may have a different relationship to depressive symptoms in the two studies. To test this, we conducted regressions with risk criterion by study interaction terms, as reported in Table 3.

For all individuals, there were significant interaction terms with the following risk criteria: familial psychiatric illness, negative emotionality and chronic difficulties. For family psychiatric illness, this interaction was due to no difference in depressive symptoms between those with affected relatives and those without in the CHAMP study. Within the ROOTS study, individuals with familial

Table 1 Proportions of individuals exhibiting each risk criterion in the CHAMP and the ROOTS study (table shows the full sample and those considered at high risk)

\begin{tabular}{|c|c|c|c|c|c|}
\hline \multirow[t]{2}{*}{ Risk criterion } & \multicolumn{2}{|c|}{$\begin{array}{l}\text { CHAMP study ( } n=1,300 \text { full sample } \\
\text { and } n=692 \text { high risk) }\end{array}$} & \multicolumn{2}{|c|}{$\begin{array}{l}\text { ROOTS study ( } n=1,144 \text { full } \\
\text { sample and } n=409 \text { high risk) }\end{array}$} & \multirow[t]{2}{*}{ Sig.* } \\
\hline & Present & Absent & Present & Absent & \\
\hline \multicolumn{6}{|c|}{ Family psychiatric illness } \\
\hline Full & $27.9 \%(362)$ & $72.1 \%(937)$ & $20.3 \%(227)$ & $79.7 \%(892)$ & $<.001$ \\
\hline High risk & $52.3 \%(362)$ & $47.7 \%(330)$ & $56.8 \%(227)$ & $43.3 \%(173)$ & .16 \\
\hline \multicolumn{6}{|l|}{ Life events } \\
\hline Full & $25.6 \%(296)$ & $74.4 \%(859)$ & $7.3 \%(81)$ & $92.7 \%(1,027)$ & $<.001$ \\
\hline High risk & $36.6 \%(233)$ & $63.4 \%(404)$ & $16.0 \%(63)$ & $84.0 \%(330)$ & $<.001$ \\
\hline \multicolumn{6}{|c|}{ Marital problems } \\
\hline Full & $30.2 \%(392)$ & $69.9 \%(908)$ & $46.8 \%(532)$ & $53.2 \%(604)$ & $<.001$ \\
\hline High risk & $49.7 \%(344)$ & $50.3 \%(348)$ & $75.8 \%(304)$ & $24.2 \%(97)$ & $<.001$ \\
\hline \multicolumn{6}{|l|}{ Bereavements } \\
\hline Full & $32.2 \%(416)$ & $67.9 \%(878)$ & $5.3 \%(57)$ & $94.7 \%(1,023)$ & $<.001$ \\
\hline High risk & $51.2 \%(352)$ & $48.8 \%(336)$ & $10.9 \%(41)$ & $89.2 \%(337)$ & $<.001$ \\
\hline \multicolumn{6}{|c|}{ Negative emotionality } \\
\hline Full & $21.9 \%(282)$ & $78.1 \%(1,008)$ & $13.1 \%(146)$ & $87.0 \%(971)$ & $<.001$ \\
\hline High risk & $30.6 \%(210)$ & $69.4 \%(476)$ & $26.7 \%(106)$ & $73.3 \%(291)$ & .17 \\
\hline \multicolumn{6}{|c|}{ Chronic difficulties } \\
\hline Full & $13.7 \%(177)$ & $86.3 \%(1,119)$ & $22.1 \%(246)$ & $77.9 \%(868)$ & $<.001$ \\
\hline High risk & $19.4 \%(134)$ & $80.6 \%(556)$ & $46.2 \%(182)$ & $53.8 \%(212)$ & $<.001$ \\
\hline \multicolumn{6}{|l|}{ Risk status } \\
\hline Full & $53.2 \%(692)$ & $46.8 \%(608)$ & $35.8 \%(409)$ & $64.3 \%(735)$ & $<.001$ \\
\hline
\end{tabular}

* Study main effect from logistic regression tests 
Table 2 Means (and SD) of the MFQ total scores for the CHAMP and ROOTS studies for the full sample and the high and low risk samples only

\begin{tabular}{llll}
\hline & CHAMP $\left(n=500^{\mathrm{a}}\right)$ & ROOTS $(n=1,089)$ & Sig.* \\
\hline MFQ, full sample & $17.1(10.6)$ & $15.2(9.9)$ & $<.001$ \\
MFQ, high risk only & $17.8(11.0)(n=394)$ & $18.0(11.1)(n=380)$ & .71 \\
MFQ, low risk only & $14.6(8.7)(n=106)$ & $13.7(8.9)(n=709)$ & .24 \\
\hline
\end{tabular}

* Significance of the study main effect while controlling for gender differences

${ }^{a} \mathrm{~N}$ number has decreased as the MFQ was only administered to individuals who consented to take part in the yearlong study

Table 3 Means (and SD) of the MFQ total scores for the CHAMP and ROOTS studies by the presence or absence of each individual risk criterion for the full sample and high risk individuals only

\begin{tabular}{|c|c|c|c|c|c|}
\hline \multirow[t]{2}{*}{ Risk criterion } & \multicolumn{2}{|c|}{ CHAMP ( $n=500$ full sample and $n=394$ high risk $^{\mathrm{a}}$ ) } & \multicolumn{2}{|c|}{ ROOTS ( $n=1,089$ full sample and $n=378$ high risk $^{\mathrm{a}}$ ) } & \multirow[t]{2}{*}{ Sig.* } \\
\hline & Present & Absent & Present & Absent & \\
\hline \multicolumn{6}{|c|}{ Family psychiatric illness } \\
\hline Full & $17.0(10.4)$ & $17.1(10.8)$ & $17.5(10.9)$ & $14.5(9.5)$ & .02 \\
\hline High risk & $17.0(10.4)$ & $18.7(11.7)$ & $17.5(10.9)$ & $17.9(11.0)$ & .52 \\
\hline \multicolumn{6}{|l|}{ Life events } \\
\hline Full & $20.7(11.6)$ & $15.7(9.8)$ & $21.9(13.0)$ & $14.7(9.5)$ & .32 \\
\hline High risk & $21.1(11.8)$ & $16.1(10.1)$ & $22.8(14.0)$ & $17.1(10.3)$ & .80 \\
\hline \multicolumn{6}{|c|}{ Marital problems } \\
\hline Full & $19.0(11.3)$ & $15.8(10.0)$ & $16.7(10.3)$ & $13.9(9.4)$ & .82 \\
\hline High risk & $19.1(11.4)$ & $16.6(10.5)$ & $18.4(11.0)$ & $16.7(11.6)$ & .49 \\
\hline \multicolumn{6}{|l|}{ Bereavements } \\
\hline Full & $18.8(11.8)$ & $15.9(9.6)$ & $19.1(12.7)$ & $15.0(9.8)$ & .65 \\
\hline High risk & $19.2(11.9)$ & $16.4(9.9)$ & $21.6(12.4)$ & $17.7(11.0)$ & .78 \\
\hline \multicolumn{6}{|c|}{ Negative emotionality } \\
\hline Full & $18.9(11.9)$ & $16.4(10.0)$ & $20.0(10.7)$ & $14.4(9.5)$ & .05 \\
\hline High risk & $19.1(12.0)$ & $17.2(10.5)$ & $21.7(10.9)$ & $16.2(10.5)$ & .07 \\
\hline \multicolumn{6}{|c|}{ Chronic difficulties } \\
\hline Full & $15.6(9.6)$ & $17.4(10.8)$ & $17.0(11.2)$ & $14.7(9.4)$ & .01 \\
\hline High risk & $15.7(9.7)$ & $18.3(11.2)$ & $17.8(11.3)$ & $17.9(10.8)$ & .25 \\
\hline
\end{tabular}

* Significance of interaction term while controlling for gender differences in linear regression

${ }^{a}$ Number of individuals within each combination differed slightly depending availability of the risk criterion variable

psychiatric illness had increased levels of depressive symptoms. There were increased depressive symptoms in those with high negative emotionality scores for both samples. In CHAMP those not exposed to chronic difficulties had more depressive symptoms, whereas the expected pattern was observed in the ROOTS study.

The results were replicated with only high risk individuals from both studies and no significant interaction terms were found between the individual risk criteria and the study variable. Overall, the relationship between depressive symptoms and the individual risk criteria was not significantly different for high risk participants in the CHAMP and ROOTS studies.

Thus, the studies have a sufficiently similar association between risk criteria and depressive symptoms.
Longitudinal trajectory of depressive symptoms

Combining the samples allowed us to use both cross sectional and longitudinal data to investigate the trajectory of depressive symptoms. Within the CHAMP high risk study, each age treated as a distinct age cohort. For reference, previous research has found that there is good specificity at a cut-off of 29 on MFQ for differentiating individuals a major depressive disorder [27].

Figure 1 displays the average total score of depressive symptoms by each age group at baseline for the CHAMP study and the longitudinal trajectory of the ROOTS study. Within the CHAMP study, there were no age differences in depressive symptoms for the female participants, $p s>.3$. For the male participants, there was a significant decrease 
Fig. 1 Average total score for depressive symptoms at age 12 , 13, 14 and 15 CHAMP cohorts $(n=868)$ and the longitudinal trajectories in the ROOTS sample $(n=1,181)$ for female and male participants

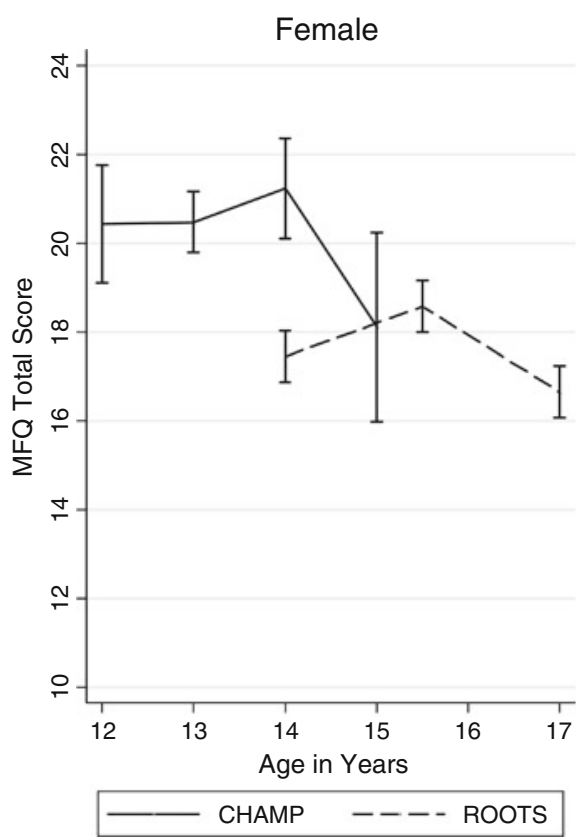

of between 2.5 to just over 4.5 points on the MFQ from age 12 to ages 13,14 and $15, \beta=-2.53,95 \%$ CI $(-4.69$, $-.37), \quad p<.05 ; \beta=-3.11,95 \%$ CI $(-5.53,-.68)$, $p<.05$; and $\beta=-4.63,95 \%$ CI $(-7.84,-1.41), p<.01$, respectively. There were no other differences between the age cohorts, $p s>.1$.

We analysed differences between the genders in each age cohort separately. There was no significant difference between the genders at age $12, p=.71$. At age 13 and 14 , females had significantly more depressive symptoms than males, $\beta=3.22$, 95\% CI $(1.45,5.00), p<.001$ and $\beta=4.56,95 \%$ CI $(2.05,7.08), p<.001$, respectively. However, there was no difference at age $15, p=.10$, where the sample size was smaller.

Within the ROOTS epidemiological study, there were significant linear and quadratic differences from age 14 to $17, \beta=1.85,95 \% \mathrm{CI}(.55,3.16), p<.01$ and $\beta=$ $-.71,95 \%$ CI $(-1.15,-.28), p<.005$, respectively. When tested categorically, it was found that depressive symptoms were higher at age 15.5 than at either age 14 or $17, \beta=1.18,95 \%$ CI $(.14,2.22), p<.05$ and $\beta=2.02$, 95\% CI $(.86,3.18), p<.005$, respectively. There was no difference between 14 and 17 years, $p>.10$. The pattern of results differed for male participants, with no significant linear and quadratic trends from age 14 to $17, p s>.5$.

Elevated levels of depressive symptoms were found in female compared to male ROOTS participants, $\beta=4.48$, $95 \%$ CI $(3.42,5.54), p<.001$. This was found to be independent of time point, as the interaction term between age and gender was not significant, $p=.85$.
The results when only looking at the CHAMP male and female high risk individuals were replicated from the full sample analysis and are shown in Fig. 2.

Within the ROOTS study, there were no linear or quadratic differences in high risk female depressive symptoms between the ages of 14 and 17, $p \mathrm{~s}>.09$, which contrasts with the quadratic differences when considering the full sample shown in Fig. 1. The results for the male ROOTS high risk individuals were replicated from the full sample as were the gender differences in both studies.

\section{Discussion}

There was overall a striking similarity in the pattern of results when looking at the high risk individuals across the two studies. The main hypothesis was supported in demonstrating no significant differences between studies when looking at risk status and depression scores.

There were differences between studies for participants exposed to familial psychiatric illness. Similar levels of depressive symptoms were present in the CHAMP high risk study for those exposed and not exposed to this risk. This was likely due to higher prevalence of other risk criteria in the CHAMP study when familial psychiatric illness was absent.

The difference within the chronic difficulties risk criterion was unexpected, in that there were more problems in those without chronic difficulties in CHAMP. The expected pattern of higher depressive symptoms associated with chronic difficulties was found within ROOTS. This may 
Fig. 2 Average MFQ total score for age $12,13,14$ and 15 CHAMP cohorts $(n=678)$ and the longitudinal trajectories in the ROOTS study $(n=399)$ for high risk female and male participants

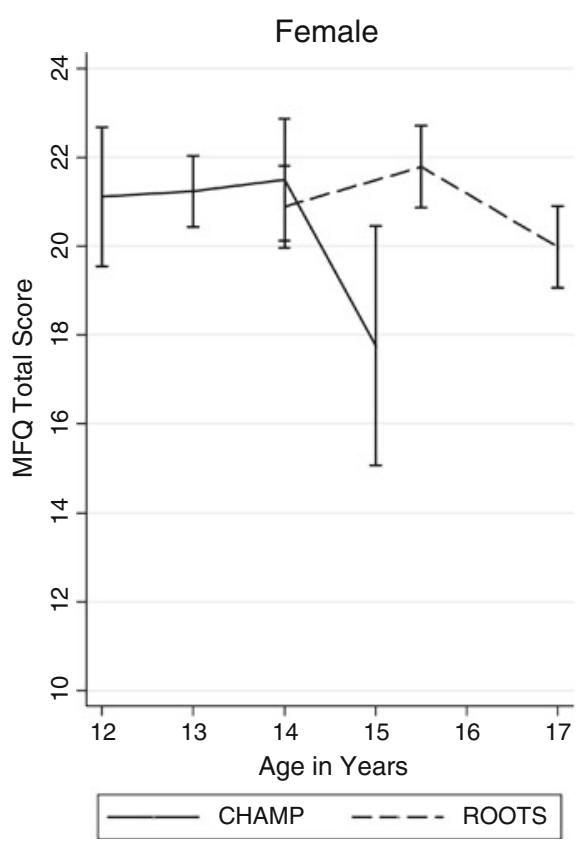

relate to a lower prevalence of chronic difficulties in the CHAMP study, perhaps due to less sensitive measurement. Due to the lower prevalence, this risk criterion was less frequently used to establish high risk status in the CHAMP study, which indicates increased levels of the remaining risk factors.

When looking at just high risk individuals, there were no differences in depressive symptoms for any of the risk criteria across the two studies. Thus, the results provide convincing evidence that the high risk CHAMP participants selected on multiple risk criteria are fundamentally similar to individuals with the same risk profiles found within an epidemiological sample.

The trajectories of depressive symptoms in the two samples when including all individuals showed substantial overlap. The CHAMP study had higher rates of depressive symptoms than the ROOTS epidemiological study, which is consistent with the expected sampling differences between the studies. Depressive symptoms in females generally remained high or increased slightly in early to mid adolescence then decreased in later adolescence. Depressive symptoms in male participants decreased in early adolescence, with a relatively consistent level for the remainder of adolescence. The emergence of a gender difference in depressive symptoms from age 13 onwards is consistent with previous reports [25, 26], and potentially linked to the onset of menarche in females [28], probably indicating an earlier rate of maturation overall.

A similar pattern of depressive symptom trajectories was found within only high risk male participants, at only a slightly elevated level of depressive symptoms. However, the pattern for female high risk individuals differed in that there was an elevated rate of depressive symptoms from age 12 onwards, and there was no longer a significant decrease in later adolescence. There was also greater overlap in the trajectories of depressive symptoms in high risk females across CHAMP and ROOTS. To a lesser extent, a similar pattern was seen across the two studies in high risk males. Overall, these results indicate recent stressful life events may not adversely influence male as much as female adolescents [26].

\section{Limitations}

Ethical rules for study participation were markedly different between the two studies. CHAMP individuals were selected on an 'opt out' whereas ROOTS operated on an 'opt in' model. There is little doubt that such variation in sample ascertainment alters the likelihood of individual participation between the two studies. This may account for higher rates of familial psychiatric illness, negative emotional temperaments, more bereavements and negative life events in the CHAMP compared to the ROOTS study. When isolating only high risk individuals, there were similar rates of familial psychiatric illness and negative emotionality but the remaining prevalence differences remained elevated within the CHAMP study.

Only two of the risk criteria, life events and negative emotionality, used the same measurement across both studies, but highly similar methods were used for the familial psychiatric illness risk criterion. The ROOTS study collected much more detailed information on marital problems, bereavements and chronic difficulties over the life course of the probands than did the CHAMP study. Therefore, it is not 
surprising that there were higher rates of marital problems and chronic difficulties within the ROOTS study.

\section{Conclusions}

Screening individuals based on risk criteria isolate a similar subset of high risk individuals found in epidemiological sampling. Depressive symptoms are maintained at a high level through the earlier period of adolescent development for all female participants then may decrease from mid to late adolescence. Female adolescents at high risk, however, are likely have higher and more stable levels of depressive symptoms from early to late adolescence than females in the population at large. Male participants are likely to have higher levels of depressive symptoms earlier in development which then decrease to a relatively consistent level throughout adolescence. This male pattern was independent of risk status. During adolescence, males may be less sensitive than females when exposed to an equivalent level of recent adverse life experiences.

Acknowledgments This work was supported by a Wellcome Trust programme grant awarded to I.M.G. and J.H. for the CHAMP data, a Wellcome Trust programme grant (grant no. 74296) for the ROOTS data collection awarded to I.M.G. J.H. P.B.J. and T.C., and the NIHR Collaboration for Leadership in Applied Health Research and Care (CLAHRC) for Cambridgeshire and Peterborough for data analysis and manuscript preparation. T.C.s contribution was part-supported by a Department of Health Career Scientist Award (Public Mental Health).

Conflict of interest The authors declare that they have no conflict of interest.

Open Access This article is distributed under the terms of the Creative Commons Attribution Noncommercial License which permits any noncommercial use, distribution, and reproduction in any medium, provided the original author(s) and source are credited.

\section{References}

1. Ingram RE, Ritter J (2000) Vulnerability to depression: cognitive reactivity and parental bonding in high-risk individuals. J Abnorm Psychol 109:588-596

2. Angold A, Weissman MM, John K, Merikangas KR, Prusoff BA, Wickramaratne P, Gammon GD, Warner V (1987) Parent and child reports of depressive symptoms in children at low and high risk of depression. J Child Psychol Psychiatry 28:901-915

3. Biederman J, Faraone SV, Hirshfeld-Becker DR, Friedman D, Robin JA, Rosenbaum JF (2001) Patterns of psychopathology and dysfunction in high-risk children of parents with panic disorder and major depression. Am J Psychiatry 158:49-57

4. Weissman MM, Wickramaratne P, Nomura Y, Warner V, Verdeli H, Pilowsky DJ, Grillon C, Bruder G (2005) Families at high and low risk for depression: a three-generation study. Biol Psychiatry 57:953-960

5. Avenevoli S, Stolar M, Li J, Dierker L, Ries Merikangas K (2001) Comorbidity of depression in children and adolescents: models and evidence from a prospective high-risk family study. Biol Psychiatry 49:1071-1081

6. Appleyard K, Egeland B, van Dulmen MHM, Sroufe LA (2005) When more is not better: the role of cumulative risk in child behavior outcomes. J Child Psychol Psychiatry 46:235-245

7. Walsh SD, Levine SZ, Levav I (2011) The association between depression and parental ethnic affliation and socioeconomic stauts: a 27-year longitudinal US community study. Soc Psychiatry Psychiatr Epidemiol (in press)

8. Clavarino A, Hayatbakhsh MR, Williams GM, Bor W, O'Callaghan M, Najman JM (2011) Depression following marital problems: Different impacts on mothers and their children? A 21-year prospective study. Soc Psychiatry Psychiatr Epidemiol 46: 833-841

9. Alloy LB, Abramson LY, Hogan ME, Whitehouse WG, Rose DT, Robinson MS, Kim RS, Lapkin JB (2000) The Temple-Wisconsin Cognitive Vulnerability to Depression Project: lifetime history of Axis I psychopathology in individuals at high and low cognitive risk for depression. J Abnorm Psychol 109:403-418

10. Dunn V, Abbot RA, Croudace T, Wilkinson P, Jones PB, Herbert J, Goodyer IM (2011) Profiles of family-focused adverse experiences through childhood and early adolescence: The ROOTS Project a community investigation of adolescent mental health. BMC Psychiatry 11:109.http://www.biomedcentral.com/14711244X/1411/1109

11. Green JG, McLaughlin KA, Berglund PA, Gruber MJ, Sampson NA, Zaslavsky AM, Kessler RC (2010) Childhood adversities and adult psychiatric disorders in the national comorbidity survey replication I: associations with first onset of DSM-IV disorders. Arch Gen Psychiatry 67:113-123

12. Rutter M (1979) Protective factors in children's responses to stress and disadvantage. In: Kent MW, Rolf JE (eds) Primary prevention of psychopathology: social competence in children, vol 3. University of New England Press, Hanover, pp 49-74

13. Sameroff AJ (2000) Developmental systems and psychopathology. Dev Psychopathol 12:297-312

14. Goodyer IM, Herbert J, Tamplin A, Altham PM (2000) Recent life events, cortisol, dehydroepiandrosterone and the onset of major depression in high-risk adolescents. $\mathrm{Br} \mathrm{J}$ Psychiatry 177:499-504

15. Goodyer IM, Herbert J, Tamplin A, Altham PM (2000) Firstepisode major depression in adolescents. Affective, cognitive and endocrine characteristics of risk status and predictors of onset. $\mathrm{Br}$ J Psychiatry 176:142-149

16. Goodyer IM, Bacon A, Ban M, Croudace T, Herbert J (2009) Serotonin transporter genotype, morning cortisol and subsequent depression in adolescents. Br J Psychiatry 195:39-45

17. Goodyer IM, Croudace T, Dudbridge F, Ban M, Herbert J (2010) Polymorphisms in BDNF (Val66Met) and 5-HTTLPR, morning cortisol and subsequent depression in at-risk adolescents. $\mathrm{Br} \mathbf{J}$ Psychiatry 197:365-371

18. Goodyer IM, Croudace T, Dunn V, Herbert J, Jones PB (2010) Cohort profile: risk patterns and processes for psychopathology emerging during adolescence: the ROOTS project. Int $\mathrm{J}$ Epidemiol 39:361-369

19. Costello EJ, Angold A (1988) Scales to assess child and adolescent depression: checklists, screens, and nets. J Am Acad Child Adolesc Psychiatry 27:726-737

20. Kent L, Vostanis P, Feehan C (1997) Detection of major and minor depression in children and adolescents: evaluation of the Mood and Feelings Questionnaire. J Child Psychol Psychiatry 38:565-573

21. Angold A, Costello E, Messer SC, Pickles A, Winder F, Silver D (1995) The development of a short questionnaire for use in epidemiological studies of depression in children and adolescents. Int J Methods Psychiatr Res 5:237-349 
22. Mathiesen KS, Tambs K (1999) The EAS temperament questionnaire-factor structure, age trends, reliability, and stability in a Norwegian sample. J Child Psychol Psychiatry 40:431-439

23. Kelvin RG, Goodyer IM, Teasdale JD, Brechin D (1999) Latent negative self-schema and high emotionality in well adolescents at risk for psychopathology. J Child Psychol Psychiatry 40:959-968

24. StataCorp (2009) Stata Statistical Software: Release 11. StataCorp LP, College Station, TX

25. Ge X, Conger RD, Elder GH Jr (2001) Pubertal transition, stressful life events, and the emergence of gender differences in adolescent depressive symptoms. Dev Psychol 37:404-417
26. Ge X, Lorenz FO, Conger RD, Elder GH (1994) Trajectories of stressful life events and depressive symptoms during adolescence. Dev Psychol 30:467-483

27. Daviss WB, Birmaher B, Melhem NA, Axelson DA, Michaels SM, Brent DA (2006) Criterion validity of the Mood and Feelings Questionnaire for depressive episodes in a clinic and non-clinic subjects. J Child Psychol Psychiatry 47:927-934

28. Joinson C, Heron J, Lewis G, Croudace T, Araya R (2011) Timing of menarche and depressive symptoms in adolescent girls from a UK cohort. Br J Psychiatry 198:17-23 